\title{
A rare case of a pituitary tumour with orbital invasion and moderate proptosis
}

Dr Gurm it Gill1, Mr Shahzada Ahmed2, Miriam As ia1, Dr John Ayuk1, Dr Niki Karavataki1, Profes sor Ne il Gittoes 1 Queen Elizabeth Hospital, Birmingham. Centre for Endocrinology, Diabetes and Metabolism1 \& Department of Otolaryngology2

\section{Case Background}

A $61 \mathrm{~F}$ without significant past medical history presented to her optician in February 2018 with clouding of vision and a moderate left sided proptosis. Ophthalmic examination revealed vision of 6/7.5 in the right eye and 6/9.5 in the left eye. She also demonstrated diplopia on upward and right lateral gaze.

An urgent brain MRI revealed a $6.3 \times 5.6 \times 5.8 \mathrm{~cm}$ mass lesion centred in the clivus and pituitary fossa, expanding in all directions. The bulk of the lesion was in the left parasellar region, encasing a patent cavernous segment of the internal carotid artery, displacing the left arm of the circle of Willis superiorly. Anteriorly on the left side, the lesion was insinuating through the left superior orbital fissure and optic canal, displacing and partially encasing the left optic nerve medially with a small component protruding into the left orbital component and causing moderate proptosis. Posteriorly the lesion was plastered against the surface of midbrain and pons and partially encased the basilar artery.

Baseline pituitary function was normal and a transsphenoidal biopsy in March 2018 was consistent with a pituitary adenoma, with negative hormone staining and focally increased Ki67 ( 6$7 \%)$.

Transsphenoidal debulking was performed in May 2018 and pathology confirmed the previous findings with Ki-67 10\%. Postoperative assessment thus far reveals unchanged vision and residual left-sided proptosis.
MRI Images
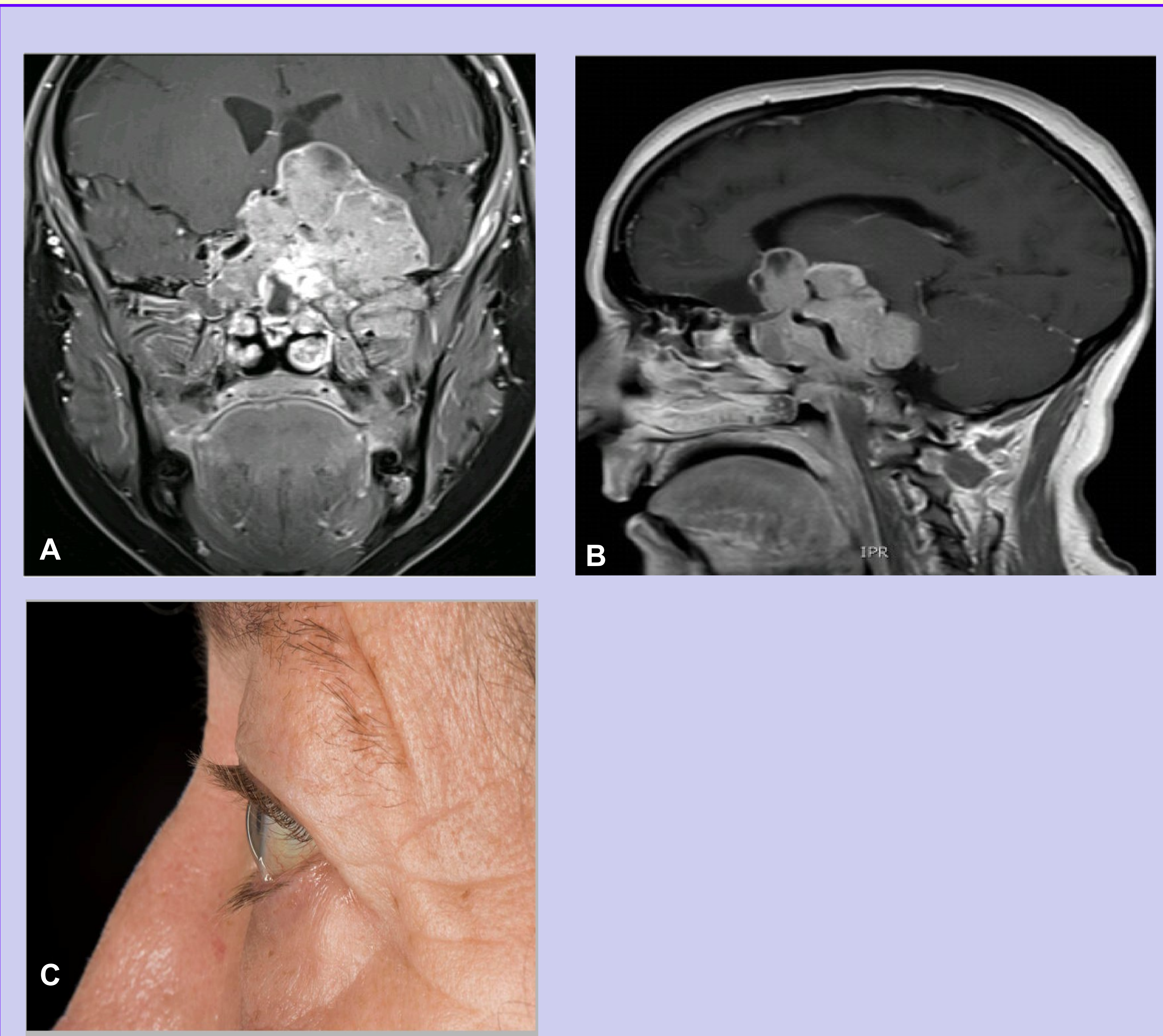

A) Coronal \& B) Sagital Pre-operative MRI sequences taken April 2018 demonstrating $6.3 \times 5.6 \times 5.8 \mathrm{~cm}$ mass in the pituitary fossa encasing the internal carotid artery with protrusion into the left orbital component causing moderate

C) Clinical Photography demonstrating moderate left sided proptosis.

\section{Case follow up}

- Post operative review revealed a surgical cavity with significant residual skull base tumour and small amount resected transphenoidally but with no convincing interval change in comparison to that of the pre-operative imaging.

- This difficult case was subsequently discussed at MDT. It was felt that further surgery would not be without significant morbidity. Consideration was made for medical therapy and the patient was subsequently commenced on somatostatin analogue in an attempt to control tumour progression.

- At latest clinic review, hormonal evaluation remains normal and she is tolerating the somatostatin analogue, apart from a few gastrointestinal symptoms. The plan is for a further MRI in January 2019 alongside further clinical evaluation and follow up. She continues to demonstrate mild proptosis on the left of $3-4 \mathrm{~mm}$.

\section{Conclusions}

- We present a rare case of a pituitary tumour with orbital invasion and moderate left sided proptosis.

- Tumours that invade the orbit are uncommon, usually meningiomas, rarely pituitary adenomas (66\% vs < 5\% respectively). 4 of only 22 orbitally invasive pituitary adenoma published case reports described similar non-functional adenoma at histology as in this case.

- Surgical resection, after excluding hormonal hypersecretion, is the optimal treatment choice for invasive pituitary tumours. However, technical difficulty due to the close proximity of the optic nerve and surrounding structures may lend to incomplete resection. Radiotherapy postoperatively may be considered depending on residual tumour burden. Post-operative somatostatin analogue use may offer limited tumour size reduction.

- The prognosis of pituitary tumours with orbital invasion is considered poor, depending significantly on histology, extent of invasion and tumour burden.

\section{References}

1) J orbensen M, Heegaard S. A review of nasal,paranasal, and skull base tumours invading the orbit. Surv Opthalmol 2017;63:389- 405

2) Albert DM, Ni C, Sebag J, Renna T. Rare orbital tumours. Int Opthalmol Clin. 1982;22(1):183-205

3) Wray SH. Neurophthalmic manifestations of pituitary and parasellar lesions. Clin Neurosurg. 1977;24:86-117

4) Naquib MM, Mendoza PR, Jariyakosal S, Grossniklaus HE. Atypical pituitary adenoma with orbital invasion: Case report and review of the literature. Surv Ophthalmol 2017;62(6):867-74 